\title{
RDUS
}

Revue de DROIT

UNIVERSITÉ DE SHERBROOKE

Titre : $\quad$ LA CONDAMNATION DE L'ASSUREUR À PAYER À SON ASSURÉ LES FRAIS EXTRAJUDICIAIRES ENCOURUS SUR L'ACTION EN GARANTIE ACCUEILLIE

Auteur(s) : $\quad$ Mark D. BANDRAUK

Revue : $\quad$ RDUS, 1992-1993, volume 23, numéro 1

Pages: $\quad 157-171$

ISSN : $\quad 0317-9656$

Éditeur : $\quad$ Université de Sherbrooke. Faculté de droit.

URI : $\quad$ http://hdl.handle.net/11143/13383

DOI : $\quad$ https://doi.org/10.17118/11143/13383 
Page vide laissée intentionnellement. 


\section{LA CONDAMNATION DE L'ASSUREUR À PAYER À SON ASSURÉ LES FRAIS EXTRAJUDICIAIRES ENCOURUS SUR L'ACTION EN GARANTIE ACCUEILLIE}

par Mark D. BANDRAUK*

Le présent article constitue une analyse approfondie des principes proposés par les autorités tant doctrinales que jurisprudentielles au sujet de la possibilité pour l'assuré de réclamer de son assureur le remboursement des frais extrajudiciaires qu'il a encourus par suite du refus de couverture de son assureur.

L'article aborde plus particulièrement le recours approprié pour l'assuré qui désire obtenir un tel dédommagement ainsi que la distinction qu'il est possible de faire entre les frais extrajudiciaires encourus par l'assuré pour se défendre à l'encontre de l'action principale et les frais extrajudiciaires qu'il a encourus sur l'action en garantie contre son assureur.

This article is intended to provide an in-depth analysis of the principles proposed both by the case law and the doctrinal authorities concerning the possibility for an insured to obtain payment from his insurer of the legal fees incurred by the former following a coverage refusal by the latter.

The article examines more specifically the proper recourse available to an insured in such a situation as well as the possible distinction to be made between the legal fees incurred by the insured in order to defend himself in the principal suit and the legal fees incurred in the warranty suit against his insurer.

* Avocat, Fournier Demers. Les recherches concernant cet article s'arrêtent au 1er janvier 1993. 


\section{SOMMAIRE}

A. La restriction des «frais et dépens» aux seuls frais judiciaires ................................ 159

B. L'extension des «frais et dépens» aux frais extrajudiciaires

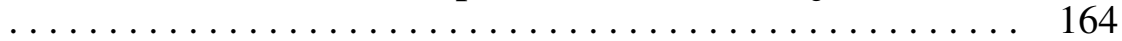

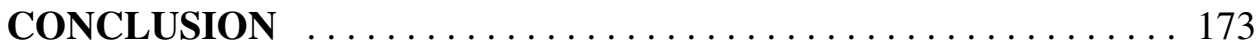


L'existence du phénomène de l'appel en garantie en matière d'assurance est loin d'être nouveau mais les conséquences possibles pour l'assureur à la suite d'un refus de couverture injustifié à l'égard de son assuré faisaient partie, jusqu'à récemment, d'un sujet sur lequel les autorités tant doctrinales que jurisprudentielles n'avaient pas eu l'occasion de se prononcer.

Toutefois, depuis une quinzaine d'années, bon nombre de ces autorités ont émis diverses opinions sur la possibilité pour un tribunal de condamner un assureur qui avait refusé de prendre le «fait et cause» pour l'assuré, de lui payer les frais extrajudiciaires encourus par celui-ci pour se défendre, que ce soit à la suite du manquement par l'assuré de l'obligation de donner l'avis de sinistre sans délai, selon l'article 2572 C.c.B.C., ou encore dans l'hypothèse où l'assureur prétend que le sinistre n'est pas couvert d'après le libellé même des clauses de la police d'assurance.

L'assuré a commencé à réclamer des honoraires extrajudiciaires contre son assureur lorsqu'il refusait de le couvrir à la suite de l'adoption de l'article 2605 C.c.B.C. en 1974 et qui se lit ainsi:

Les frais et dépens des poursuites contre l'assuré, y compris ceux de la défense et des intérêts sur le montant de la garantie, sont à la charge de l'assureur en sus du montant des assurances.

Cette disposition a donné naissance à deux tendances que nous allons examiner, soit la restriction des «frais et dépens» aux seuls frais judiciaires et l'extension des «frais et dépens» aux frais extrajudiciaires.

\section{A. La restriction des «frais et dépens» aux seuls frais judiciaires}

L'auteur Alain Létourneau interprète de la manière suivante l'article 2605 C.c.B.c. en ce qui a trait aux «frais et dépens»:

Donc ici, la couverture recherchée n'est pas seulement l'indemnisation de toute condamnation mais inclut l'obligation pour l'assureur de prendre les faits et causes de la partie et d'assumer la défense. Ceci laisse croire que, lorsque, à l'article suivant on parle de frais et dépens, le législateur a peut-être voulu inclure les frais extrajudiciaires que l'assureur aurait encouru nécessairement, eut-il assumé la défense comme il le devait; bien entendu, si on peut prouver qu'il le devait. C'est là, évidemment une hypothèse car, comme la loi parle toujours, on doit donc donner un sens à l'article 2605 qui parle expressément de frais et dépens des poursuites contre l'assuré, ce qui ne peut certainement inclure plus que les frais taxables et parle ensuite de "ceux de la défense" qui doivent donc être de même nature 
que ceux de la poursuite et, dans un tel cas, se limiterait également aux frais taxables ${ }^{1}$.

Le professeur Didier Lluelles affirme dans le même sens:

L'assureur tenu d'assumer la défense de l'assuré doit impérativement prendre à sa charge les frais et dépens des poursuites contre l'assuré, tant en demande qu'en défense, ainsi que les intérêts (C.c. 2605: O.P.A.).

Toutefois, l'assureur ne serait pas tenu de rembourser les honoraires extrajudiciaires qu'a payés l'assuré au niveau de l'appel en garantie de l'assureur ${ }^{2}$.

Pour justifier son raisonnement sur le sort des frais extrajudiciaires réclamés par l'assuré dans le cas d'un appel en garantie contre un assureur, le professeur Lluelles s'appuie sur les considérants de la cause Paquin c. Cie d'Assurances générales de commerce ${ }^{3}$. Dans cette affaire, le tribunal était saisi d'une action en réclamation d'honoraires extrajudiciaires à l'encontre de l'assureur qui avait refusé de prendre le fait et cause de l'assuré.

La Cour supérieure, dont le jugement a été confirmé par la Cour d'appel, avait donné raison au demandeur Paquin en accueillant son appel en garantie à l'encontre de l'assureur, l'obligeant ainsi à prendre fait et cause pour l'assuré. Le juge Gauthier invoque les motifs suivants pour justifier le rejet de l'action du demandeur:

Le demandeur en poursuivant ses assureurs par appel en garantie a exercé un droit que la loi lui accorde.

Par contre, la défenderesse en refusant de défendre le demandeur, défendeur dans la cause portant le numéro 23-475 de la Cour supérieure de Joliette et dans le dossier numéro 09-000019-73 de la Cour d'Appel, district de Montréal, a elle aussi exercé un droit. Même si la Cour supérieure, confirmée en cela par la Cour d'Appel, a décidé qu'elle avait tort, à nulle part dans la jurisprudence ne retrouve-t-on des jugements condamnant une partie à payer les frais extrajudiciaires de l'autre partie ${ }^{4}$.

1. «De l'assurance de dommages sous la nouvelle loi sur les assurances» dans La nouvelle loi sur les assurances: profonds changements au C.C. et aux lois connexes, Montréal, C.F.P.B.Q., 1975, pp. 141 et 142.

2. Didier LLUELLES, Droit des assurances - Aspects contractuels, 2e éd., Montréal, Les Éditions Thémis Inc., 1986, p. 249.

3. [1980] C.S. 296.

4. Id., p. 297. 
Notons, toutefois, que dans son dispositif, le tribunal se réfère à la police d'assurance qui spécifiait que l'obligation de l'assureur d'assumer les frais et dépens étaient limités à «tous les frais taxés contre l'Assuré dans telle poursuite contestée... », ce qui a possiblement influé sur sa décision.

Dans une affaire semblable, le tribunal s'est prononcé sur la réclamation d'honoraires extrajudiciaires ${ }^{5}$.

Dans ce litige, la compagnie d'assurance défenderesse avait refusé de prendre fait et cause pour le demandeur pour le motif que les procédures, qui avaient été intentées contre ce dernier n'entraient pas dans le cadre de la couverture offerte par la police d'assurance en question. La Cour supérieure a donné raison à la défenderesse en décidant «que les deux requêtes présentées par la Ville de Montréal-Est contre le demandeur ne l'obligeaient aucunement à prendre sa défense, car elles n'étaient pas intentées "pour un motif faisant l'objet du contrat"6».

Tout en arrivant à la conclusion que la compagnie d'assurance défenderesse était justifiée de refuser d'assumer la défense du demandeur, le tribunal ne se prononce pas sur la portée de l'obligation de l'assureur de payer les frais et dépens de son assuré selon l'article 2605 C.c.B.C.

Enfin, l'arrêt Guay c. Garage DL inc. ${ }^{7}$ confirme cette position.

Dans ce litige, la demanderesse avait intenté une action contre la défenderesse dans laquelle la compagnie d'assurance défenderesse en garantie avait refusé de prendre fait et cause pour l'assuré. L'inaction de la compagnie d'assurance a contraint la défenderesse à retenir les services d'un avocat pour négocier directement un règlement avec la demanderesse. Par la suite, par voie de déclaration en garantie amendée, Garage D.L. Inc. a réclamé de son assureur les honoraires extrajudiciaires encourus pour en arriver à ce règlement. C'est dans ce contexte que la demanderesse en garantie Garage DL Inc. prétendait que sa réclamation pour frais extrajudiciaires était bien fondée, et devait donc être accueillie.

Dans sa décision, le juge Boyer de la Cour du Québec se réfère à l'affaire Paquin $^{8}$ et motive ainsi le rejet de la réclamation de la demanderesse en garantie:

\footnotetext{
5. Labrosse c. Cie d'assurances générales Kansa Ltée, [1988] R.R.A. 186 (C.S.).

6. Id., p. 187

7. [1991] R.R.A. 396 (C.Q.).

8. Précité, note 3.
} 
Avec égards, le Tribunal ne peut partager le point de vue soutenu par la demanderesse en garantie. En refusant de prendre fait et cause, la défenderesse en garantie ne fait qu'exercer un droit que la loi lui reconnaît (articles 2585 à 2591 du Code civil). Les frais de l'action en garantie prononcés contre l'assureur sanctionneront éventuellement son refus injustifié d'assumer la défense de son assurée en outre des autres coûts prévus par l'article 2605 C.C. ${ }^{9}$.

En outre, le juge Boyer affirme également:

Il m'apparaît donc que ce que devait faire la demanderesse en garantie en l'instance était de voir à l'inscription et à l'audition de la cause le plus tôt possible afin de faire décider par le Tribunal des obligations de toutes les parties. Pour une raison peut-être fort valable, mais étrangère à la contestation judiciaire, la demanderesse en garantie a préféré régler elle-même la réclamation de la demanderesse. La défenderesse en garantie ne saurait être tenue en droit de payer ces dépenses auxquelles elle n'est pas obligée aux termes des articles 2604 et 2605 C.C.

Enfin, en ce qui a trait à la portion des honoraires extrajudiciaires supportés par la demanderesse pour l'appel en garantie, elle doit être compensée en principe par les honoraires judiciaires sur l'action. L'excédent demandé, le cas échéant, entre dans la catégorie des dépenses normalement encourues par tous les justiciables pour faire valoir, par ministère d'avocat, leurs droits au niveau judiciaire ${ }^{10}$.

Contrairement aux raisonnements élaborés dans les causes Paquin $^{11}$ et Labrosse $^{12}$, le juge Boyer fait une distinction entre les honoraires extrajudiciaires encourus par l'assuré pour se défendre et les honoraires extrajudiciaires pour appeler en garantie sa compagnie d'assurance. Cette distinction reviendra dans des décisions citées ultérieurement.

Somme toute, cette première école repose sur le principe selon lequel une partie ne pourrait se faire rembourser par la partie adverse des frais extrajudiciaires encourus pour faire valoir ses droits. Cette règle est primordiale

9. Précité, note 7, p. 397.

10. Id.

11. Précité, note 3.

12. Précité, note 5. 
(1992) 23 R.D.U.S.

La condamnation de l'assureur à payer à son assuré les frais extrajudiciaires

encourus sur l'action en garantie accueillie

et doit l'emporter sur toute autre considération. À l'opposé, on trouve la deuxième tendance que nous allons maintenant analyser.

\section{B. L'extension des «frais et dépens» aux frais extrajudiciaires}

La première décision judiciaire qui reconnaît à l'assuré la possibilité d'obtenir des frais extrajudiciaires relève de l'affaire Pelletier c. Prévoyants $d u$ Canada ${ }^{13}$. Il s'agissait dans ce cas d'une action en remboursement des sommes payées par l'assuré à la suite de deux condamnations prononcées contre lui dans le cadre d'une action en responsabilité civile, ainsi que les frais encourus pour assumer sa défense. Le demandeur justifie sa réclamation en prétendant que la compagnie d'assurance défenderesse aurait dû intervenir dans l'action en responsabilité civile dirigée contre lui et prendre son fait et cause.

La Cour supérieure a décidé que les prétentions du demandeur étaient bien fondées et a accueilli son action contre la compagnie d'assurance. Quant aux frais réclamés par le demandeur, incluant les frais extrajudiciaires, la Cour, sans motiver sa décision, conclut ainsi:

...condamne la défenderesse à rembourser le demandeur des sommes qu'il a dû encourir pour sa propre défense et qui s'élèvent à 1014,00 $\$ .^{14}$

Notons que la compagnie d'assurance demanderesse en a appelé de cette décision et que son appel a été accueilli. La Cour d'appel a jugé que le refus de couverture invoqué par l'assureur était justifié. L'aspect des frais extrajudiciaires accordés à l'assuré en première instance n'a donc pas été analysé par la Cour d'appel.

La seconde décision, Industries Guay Ltée c. Lessard ${ }^{15}$, reconnaît le bien-fondé de la réclamation par l'assuré des honoraires extrajudiciaires contre son assureur à la suite du refus injustifié de couverture de celui-ci. Il s'agissait encore une fois d'une situation où l'assuré avait appelé en garantie sa compagnie d'assurance à la suite de son refus de prendre fait et cause pour lui.

13. [1974] C.S. 302; cette décision a été portée en appel devant la Cour d'appel de Montréal no 500-09-000 670-745 et l'appel a été accueilli le 15 décembre 1977.

14. Id., p. 306

15. [1981] C. S. 685, décision portée en appel no 200-09-000 678-810 et réglée hors cour le 20 juin 1986. 
Après une analyse des clauses d'exclusion invoquées par l'assureur pour justifier son refus de couverture, le juge Dionne, étant d'avis que le refus de couverture de l'assureur était injustifié, exprime l'opinion suivante sur la réclamation des honoraires extrajudiciaires encourus par l'assurée pour se défendre:

Or, les dommages réclamés par dame Lessard étaient garantis par la police émise par Provinces-unies. Celle-ci devait prendrefait et cause pour son assurée, et, ayant fait défaut de le faire, elle devra supporter tous les frais légaux occasionnés à son assurée, y compris les frais extrajudiciaires qu'elle pourrait éventuellement être appelée à payer à ses propres procureurs. Tels frais sont, à notre avis, une conséquence directe du refus non justifié de Provinces-unies de défendre son assurée, ou de régler la poursuite intentée contre elle ${ }^{16}$.

Notons que le juge Dionne s'est appuyé sur le libellé même de la police d'assurance pour justifier, du moins en partie, son raisonnement. En effet, la police d'assurance prévoyait que l'assureur convenait «de payer tous les frais taxés contre l'assuré dans une telle poursuite, toutes les dépenses encourues par l'assureur (sic)...». Il s'ensuit que le juge Dionne a considéré que ces dépenses devaient inclure, entre autres, «les honoraires d'avocat que l'assuré se voit contraint d'encourir en vue d'obtenir, par voie légale, le respect de sa police ${ }^{17}$.

Cependant, au lieu de condamner la compagnie d'assurance au paiement des frais extrajudiciaires, le juge a préféré réserver les recours de l'assurée du fait que la conclusion pertinente à ce sujet dans l'action en garantie de l'assurée était loin d'être claire, sans compter qu'il n'avait présenté aucune preuve du compte d'honoraires. Nous devons toutefois reconnaître que la décision du juge Dionne, quoique créant un précédant, n'est pas concluante.

Enfin, dans l'affaire Dufour c. Charest ${ }^{18}$, la Cour supérieure, décida que l'action en garantie de l'assuré contre son assureur était bien fondée en faisant valoir que:

La demanderesse, ainsi, en voulant obtenir que cette Cour, par son appel en garantie réamendé, condamne les défenderesses en garantie à lui rembourser tout ce qu'elle est appelée à payer à titre d'honoraires extra-judiciaires à ses procureurs, modifie la substance $d u$ recours initial et en fait une réclamation en dommages découlant d'un bris de contrat;

16. Id., p. 692.
17.

18. C.S. Québec 200-05-005877-787, le 24 novembre 1982 (J.E. 83-1). 
Le Tribunal est d'avis qu'il ne peut dans ce dossier prononcer sur autre chose que sur les frais et dépens selon les articles 477 et suivants C.P.;

Mais, y a-t-il lieu de prononcer sur les dépens? La réponse est oui puisque d'une part, demande en est faite formellement et d'autre part, les défenderesses par leur défaut d'intervenir pour prendre fait et cause pour leur assurée, ont causé le recours même si parfois, le demandeur principal pourrait être condamné aux dépens d'un recours en garantie de son défendeur ${ }^{19} ; \ldots$

Dans ce jugement, le juge Laflamme discute des articles 477 et suivants du Code de procédure civile et réitère que l'obligation de défense, qui incombe à l'assureur et qui est codifié à l'article 2604 C.c.B.C., est primordiale et par surcroît d'ordre public ${ }^{20}$.

que:

Le juge Laflamme conclut en accueillant l'action en garantie avec dépens

Considérant dans les circonstances que la réclamation de la demanderesse en garantie en est une en dommages pour bris de contrat;

Considérant que son recours ainsi modifie la substance de son action en garantie et doit se faire par une action directe ${ }^{21}$.

La règle selon laquelle la réclamation d'honoraires extrajudiciaires de l'assuré contre son assureur devrait être demandée par action directe, et non à l'occasion d'une action en garantie, semble faire la quasi-unanimité si l'on considère les types d'actions qui ont été prises pour réclamer les frais extrajudiciaires payés par l'assuré.

Ainsi, dans la cause Kaufel Group Ltd c. Liberty Mutual Fire Insurance $\mathrm{Co}^{22}$, le juge Johnson devait décider à la suite de l'accueil d'une action en garantie de la demanderesse contre la défenderesse, du bien-fondé de l'action en remboursement d'honoraires extrajudiciaires intentée par l'assurée. Notons que

19. Id., pp. 10 et 11 du jugement.

20. Id., pp. 11 et 12 du jugement.

21. Id., p. 16 du jugement.

22. [1988] R.J.Q. 2729, Jugement porté en appel no 500-09-001205-889 et au sujet de laquelle aucune décision n'a encore été rendue à ce jour. 
la police d'assurance, dans cette affaire, prévoyait l'obligation pour l'assureur de défendre son assuré «at the cost of the Insurer» (aux frais de l'assureur), ce qui serait plus étendu que la notion de «frais et dépens» que l'on retrouve à l'article 2605 C.c.B.C. et dans les clauses habituelles des polices d'assurance.

Le juge Johnson analyse la théorie du professeur Lluelles, citée plus haut $^{23}$ et ajoute que:

The Court notes that the author is careful to limit the reference to extrajudicial fees to, qu'a payés l'assuré au niveau de l'appel en garantie de l'assureur. This would seem to leave open the question of the extrajudicial fees which the Insurer could incur to contest the principal action in the pleadings and at trial as distinct from those for the actual warranty proceedings themselves ${ }^{24}$.

Le juge Johnson semble affirmer, à l'égard de la réclamation d'honoraires extrajudiciaires d'un assuré contre un assureur dans ce cas, qu'il y aurait possiblement lieu de distinguer entre les frais extrajudiciaires encourus par l'assuré pour se défendre contre l'action principale et les honoraires extrajudiciaires encourus par l'assuré pour appeler en garantie son assureur.

L'assureur dans cette cause invoquait en défense que le refus de couverture de l'assuré était justifié et fondé sur une interprétation raisonnable de la police d'assurance et qu'aucune faute ne pouvait lui être reprochée, même si cela a obligé l'assuré à encourir des frais extrajudiciaires pour se défendre dans l'action principale.

Pour rejeter l'action de la demanderesse, le juge Johnson se réfère à la notion de «proof of fault», qui présente des similitudes avec la notion de l'abus de droit:

In other words the simple failure to fulfil the obligation is not enough, it requires proof of fault. In the present case the Court considers that it is clear from the judgment dated 2 April 1985 Exhibit D-3 that there were serious questions legitimately raised by the Defendant as to whether the claim was covered under the policy. These could only be resolved by evidently quite extensive proof made at the trial and serious consideration in the judgment. In the Court's opinion the Defendant in view of these serious doubts which it raised, and there

23. D. Lluelles, op. cit., note 2.

24. Précité, note 22, p. 2731. 
is nothing to suggest that it did so in bad faith, was entitled to protect itself and proceed in the way that it did.... ${ }^{25}$

Ces deux dernières décisions permettent de conclure que le recours d'un assuré contre un assureur pour honoraires extrajudiciaires serait de nature contractuelle, tirant sa source du lien entre les parties au contrat d'assurance.

Survint ensuite la curieuse affaire Paquin c. Normandin ${ }^{26}$. Il s'agissait d'une action en réclamation d'honoraires extrajudiciaires impayés intentée par une firme d'avocats contre la défenderesse à la suite de services juridiques rendus pour défendre celle-ci dans une action en responsabilité civile.

La compagnie d'assurance Groupe Commerce, qui assumait la défense de Normandin, avait refusé de prendre le fait et cause de cette dernière. Faisant face à cette réclamation d'honoraires extrajudiciaires impayés, la défenderesse Normandin appela de nouveau en garantie ses assureurs.

Par ailleurs, la défenderesse Normandin a contesté l'action des demandeurs en soulevant que le montant de la réclamation était grossièrement exagéré et qu'ils avaient en outre commis une faute professionnelle en ne demandant pas dans son action en garantie que les assureurs soient condamnés à payer non seulement les frais judiciaires, mais également les frais extrajudiciaires encourus à la suite de leur refus de prendre fait et cause pour la défenderesse Normandin. En dernier lieu, dans ce deuxième appel en garantie, intenté de nouveau contre ses assureurs, la défenderesse Normandin demandait de condamner son assureur à lui rembourser une somme additionnelle de 3000 \$ représentant le montant des honoraires extrajudiciaires convenu avec son nouveau procureur dans la poursuite intentée contre elle par ses ex-procureurs!

La Cour du Québec devait donc disposer de deux réclamations d'honoraires extrajudiciaires, l'une étant l'objet même de l'action principale et l'autre afférente à l'action en garantie.

Après un bref survol de la jurisprudence pertinente, la Cour du Québec donne l'interprétation suivante des articles 2604 et 2605 C.c.B.C.:

Les articles 2604 et 2605 C.C. se complètent. En imposant à l'assureur l'obligation de prendre le fait et cause de son assuré et d'assumer les frais et dépens des poursuites contre lui, l'intention du législateur est de faire assumer par l'assureur les frais extrajudiciaires

25. Id., p. 2732.

26. [1990] R.R.A. 509 (C.Q.). 
qu'il aurait dû de toute manière assumer s'il avait pris le fait et cause de son assuré. Autrement, l'assureur pourrait trop facilement se soustraire à son obligation d'ainsi prendre le fait et cause de son assuré et se libérer d'assumer les frais de poursuites ${ }^{27}$.

Par le fait même, la Cour du Québec accueillait l'action principale intentée par les demandeurs. Quant aux autres prétentions de la défenderesse Normandin, la Cour décida ce qui suit:

Le recours des demandeurs n'est donc pas prescrit et, en venant à cette conclusion, il va de soi que les demandeurs n'ont pas commis de faute en ne réclamant pas en Cour supérieure leurs frais extrajudiciaires ou en ne faisant pas une demande de réserve de recours. De toute manière, il aurait été prématuré de demander une condamnation au paiement des frais extrajudiciaires, alors qu'ils n'étaient pas liquidés. D'autre part, une réserve de recours ne saurait être attributive de droits ${ }^{28}$.

Il s'agit d'un argument additionnel pour les tenants de la thèse voulant que la réclamation d'honoraires extrajudiciaires d'un assuré contre son assureur pour refus de couverture injustifié doit se faire par voie d'action directe. En effet, au moment du procès sur l'action en garantie, ces dommages ne peuvent être liquidés, étant donné que leur montant exact ne sera déterminé avec certitude que lorsque le compte d'honoraires extrajudiciaires final sera transmis à l'assuré par les procureurs dont il a retenu les services.

Enfin, concernant la réclamation d'honoraires extrajudiciaires de la défenderesse Normandin contre son assureur, la Cour du Québec en dispose ainsi:

...l'entente intervenue entre le procureur de la demanderesse en garantie et cette dernière pour un montant de $3000 \$$ à titre de frais extrajudiciaires ne saurait lier la défenderesse en garantie. Elle n'a aucune obligation contractuelle ni légale de payer cette somme. Le principe nettement établi que la partie défaillante paie les frais judiciaires et non les frais extrajudiciaires doit prévaloir en l'instance, à défaut de dispositions contraires applicables ${ }^{29}$.

$\begin{array}{ll}\text { 27. } & \text { Id., p. } 513 . \\ 28 . & \text { Id. p. } 514 . \\ \text { 29. } & \text { Id. }\end{array}$ 
La Cour du Québec, du moins sur ce dernier point, rejoint l'opinion émise par le juge Boyer dans la cause Guay $^{30}$ citée plus haut.

Enfin on retrouve, dans le même sens, une décision récente ${ }^{31}$ dans laquelle le juge Pagé de la Cour du Québec devait statuer sur une action en réclamation des frais engagés par l'assurée pour assurer sa défense à la suite du refus de couverture que lui opposait son assureur. Le refus de couverture a été jugé mal fondé par la Cour supérieure qui était saisi du dossier à l'époque.

Tout en reconnaissant que la jurisprudence était contradictoire sur la possibilité de condamner un assureur aux honoraires extrajudiciaires de son assuré dans un tel cas, le juge arrive à la conclusion que l'action de l'assurée devait être accueillie pour les motifs suivants:

Après analyse des faits présentés en preuve, de la jurisprudence et de l'argument des procureurs, la Cour en vient à la conclusion que l'assureur a commis une faute, une erreur, en refusant à tort de défendre l'assurée. Prendre en charge la défense de l'assurée implique souvent des frais extrajudiciaires et cela s'est produit dans la défense à l'action présentée contre l'assureur en Cour Supérieure, (pièce P-4, copie du jugement dans ce dossier). L'assureur en est responsable 32 .

Notons que le juge Pagé a condamné la compagnie d'assurance défenderesse aux frais extrajudiciaires en plus des frais d'expertise encourus par l'assurée pour présenter une défense convenable.

Mentionnons également que le juge Pagé spécifie clairement que le montant de la condamnation à l'égard de l'assureur comprend autant les frais extrajudiciaires encourus par l'assurée pour se défendre sur l'action principale que ceux encourus par l'assurée pour appeler en garantie l'assureur ${ }^{33}$. Somme toute, il ne distingue plus entre ces deux catégories de frais extrajudiciaires.

Enfin, le juge Pagé soulève l'élément de la «faute prouvée» eu égard à l'assureur que le juge Johnson avait déjà invoqué dans l'affaire Kaufel Group Limited $^{34}$ précédemment citée.

\footnotetext{
30. Précité, note 7.

31. St-Nicéphore (Corp. municipale de) c. Laurentienne générale (La), compagnie d'assurances inc., [1992] R.R.A. 760 (C.Q.).

32. Id., p. 763.

33. Id., p. 762.

34. Précité, note 22.
} 
Cette approche rejoint en quelque sorte la théorie du professeur JeanGuy Bergeron qui écrit:

Quant aux frais extrajudiciaires la question est plus délicate. La coutume veut que chaque partie assume de tels frais. Dans le contexte de l'assurance de responsabilité, nous pensons que cette coutume n'a plus sa place. En effet, n'est-il pas anormal que l'assuré paie de tel frais alors qu'on lui a représenté qu'il n'y aurait aucuns frais et dépens à payer? Habituellement, on retrouve même une clause par laquelle on dégage l'assuré de toute contribution financière. Du moins faudrait-il faire supporter les frais et dépens à l'assureur si son refus de défendre constitue un abus de droit ${ }^{35}$.

\section{CONCLUSION}

À la lumière de ces hésitations jurisprudentielles, on réalise que le débat est loin d'être terminé, d'autant plus que les réclamations en matière d'assurance, donnant lieu inévitablement à des actions en garantie intentées par les assurés contre les assureurs, ne cessent d'augmenter.

Il est difficile de concilier les deux courants de pensée dont nous venons de faire état. Il ressort, toutefois, de notre analyse de la jurisprudence et de la doctrine que le recours de l'assuré désirant réclamer de son assureur les frais extrajudiciaires qu'il a encourus à la suite du refus de couverture de son assureur, doit s'exercer au moyen d'une action directe, de nature contractuelle, et ce, seulement lorsque le sort du refus de l'assureur de prendre fait et cause aura été clairement déterminé par jugement.

Par ailleurs, nous sommes d'avis que, d'un point de vue strictement juridique, la distinction entre les honoraires extrajudiciaires encourus par l'assuré pour se défendre sur l'action principale et les frais extrajudiciaires encourus par lui pour appeler en garantie son assureur reste la solution acceptable.

En effet, si le tribunal détermine que le refus de l'assureur de prendre fait et cause pour l'assuré n'était pas justifié, on devrait tout au plus condamner l'assureur aux seuls frais qu'il s'est engagé à couvrir en vertu de la police d'assurance et ce, en conformité avec l'article 2605 C.c.B.C., soit les frais judiciaires et extrajudiciaires engagés pour défendre l'assuré à l'encontre de l'action principale. 
(1992) 23 R.D.U.S.

La condamnation de l'assureur à payer à son assuré les frais extrajudiciaires

encourus sur l'action en garantie accueillie

En ce qui a trait aux frais extrajudiciaires encourus par l'assuré pour appeler en garantie son assureur, nous ne voyons pas comment l'assureur pourrait y être condamné. En effet, l'action en garantie constitue en elle-même un procès distinct de l'action principale, d'après les articles $216 \mathrm{C}$. et suivants, et demeure le recours approprié de l'assuré qui désire faire valoir ses droits à l'égard de son assureur. Dès lors, le principe général obligeant qu'une partie assume ses propres frais extrajudiciaires lorsqu'elle cherche à faire valoir ses droits, doit être respecté.

En dernier lieu, il faut souhaiter que la Cour d'appel soit bientôt saisie d'un litige portant principalement sur les frais extrajudiciaires réclamés d'un assureur ayant refusé de prendre «fait et cause» pour l'assuré et espérer qu'elle proposera une solution claire et motivée. 